\title{
Erratum to: InteGO2: a web tool for measuring and visualizing gene semantic similarities using Gene Ontology
}

Jiajie Peng ${ }^{1,4+}$, Hongxiang $\mathrm{Li}^{2 \dagger}$, Yongzhuang Liu${ }^{2}$, Liran Juan ${ }^{3}$, Qinghua Jiang ${ }^{3}$, Yadong Wang ${ }^{2 *}$ and Jin Chen ${ }^{4,5^{*}}$

\section{Erratum}

After the publication of this supplement [1] the URL http:// mlg.hit.edu.cn:8089/ceased to work and the new URL of https://mlg.hit.edu.cn/InteGO2 was created to replace it.

\footnotetext{
Author details

'School of Computer Science, Northwestern Polytechnical University, Xi'an, China. ${ }^{2}$ School of Computer Science and Technology, Harbin Institute of Technology, Harbin, China. ${ }^{3}$ School of Life Science and Technology, Harbin Institute of Technology, Harbin, China. ${ }^{4}$ Department of Energy Plant Research Laboratory, Michigan State University, East Lansing, MI 48824, USA.

${ }^{5}$ Department of Computer Science and Engineering, Michigan State University, East Lansing, Ml 48824, USA.
}

Received: 21 March 2017 Accepted: 21 March 2017

Published online: 28 March 2017

\section{Reference}

1. Peng J, Li H, Liu Y, Juan L, Jiang Q, Wang Y, et al. InteGO2: a web tool for measuring and visualizing gene semantic similarities using Gene Ontology BMC Genomics. 2016;17 Suppl 5:530. doi:10.1186/s12864-016-2828-6.

\footnotetext{
* Correspondence: ydwang@hit.edu.cn; jinchen@msu.edu

${ }^{\dagger}$ Equal contributors

${ }^{2}$ School of Computer Science and Technology, Harbin Institute of Technology, Harbin, China

${ }^{4}$ Department of Energy Plant Research Laboratory, Michigan State University, East Lansing, Ml 48824, USA
} 